Theoretical foundation of Zisman's empirical equation for wetting of liquids on solid surfaces

This article has been downloaded from IOPscience. Please scroll down to see the full text article.

2010 Eur. J. Phys. 31251

(http://iopscience.iop.org/0143-0807/31/2/001)

View the table of contents for this issue, or go to the journal homepage for more

Download details:

IP Address: 159.226.231.78

The article was downloaded on 25/03/2011 at 08:36

Please note that terms and conditions apply. 


\title{
Theoretical foundation of Zisman's empirical equation for wetting of liquids on solid surfaces
}

\author{
Ruzeng Zhu' ${ }^{1}$, Shuwen Cui and Xiaosong Wang \\ State Key Laboratory of Nonlinear Mechanics (LNM), Institute of Mechanics, Chinese Academy \\ of Sciences, Beijing, 100190, People's Republic of China \\ E-mail: zhurz@1nm.imech.ac.cn
}

Received 21 August 2009

Published 29 December 2009

Online at stacks.iop.org/EJP/31/251

\begin{abstract}
Theories of wetting of liquids on solid surfaces under the condition that van der Waals force is dominant are briefly reviewed. We show theoretically that Zisman's empirical equation for wetting of liquids on solid surfaces is a linear approximation of the Young-van der Waals equation in the wetting region, and we express the two parameters in Zisman's empirical equation in terms of the dielectric polarizabilities of the solid and liquids. The materials contained in this paper are suitable for physics teaching of wetting phenomena for undergraduate, graduate, general physicist, etc.
\end{abstract}

\section{Introduction}

Contact angle phenomena are common and important in solid-liquid-vapour interfacial phenomena, for instance, adhesives, lubricants, capillary penetration into porous media, flotation, etc [1-3].

The contact angle $\theta$ of a liquid drop on a solid surface is defined by the mechanical equilibrium of the drop under the action of three interfacial tensions: solid-vapour, $\sigma_{\mathrm{sv}}$, solidliquid, $\sigma_{\mathrm{sl}}$, and liquid-vapour, $\sigma_{\mathrm{lv}}$. This equilibrium relation is known as Young's equation [1-4]:

$$
\cos \theta=\frac{\sigma_{\mathrm{sv}}-\sigma_{\mathrm{sl}}}{\sigma_{\mathrm{lv}}}
$$

From contact angle measurements of homologous series of simple molecular liquids (where van der Waals forces are dominant) on low-energy solid (i.e. molecular solid) surfaces, Zisman $[5,6]$ gave a phenomenological linear relation

$$
\cos \theta=1+b\left(\sigma_{\mathrm{c}}-\sigma_{\mathrm{lv}}\right)
$$

1 Author to whom any correspondence should be addressed. 
for the wetting region, where $b$ and $\sigma_{\mathrm{c}}$ are constants depending on the solid and the liquid series.

Zisman's phenomenological linear relation has been used extensively in experimental studies of wetting phenomena [7-9]. However, its theoretical origin, i.e. how to deduce Zisman's equation (2) from Young's equation (1), is still unknown.

The purpose of this paper is to deduce Zisman's equation (2) theoretically. It is obvious that this theoretical problem is important in the field of wetting phenomena. However, the theoretical derivation of Zisman's equation (2) is so simple that the materials contained in this paper are suitable for physics teaching of wetting phenomena for undergraduate, graduate, general physicist, etc.

\section{Brief review of the Zisman equation for wetting}

Experimental studies $[5,10,11]$ have shown that the cosine of the contact angle $\theta$ of a simple molecular liquid on a given low-energy solid surface is a function of the surface tension $\sigma_{\mathrm{lv}}$ of the liquid, i.e.,

$$
\cos \theta=f\left(\sigma_{\mathrm{lv}}\right)
$$

as long as the liquids belong to a homologous series.

In 1952, Zisman [10] introduced the concept of critical surface tension $\sigma_{\mathrm{c}}$ to indicate the wettability of low-energy solid surfaces for a homologous series of simple molecular liquids. The definition of the critical surface tension $\sigma_{\mathrm{c}}$ is the surface tension which satisfies the condition $\cos \theta=1$, i.e.

$$
f\left(\sigma_{\mathrm{c}}\right)=\cos 0=1 .
$$

A liquid of the series with a surface tension $\sigma_{\mathrm{lv}}=\sigma_{\mathrm{c}}$ forms zero contact angle on the solid surface. Liquids of the series with a surface tension $\sigma_{\mathrm{lv}}<\sigma_{\mathrm{c}}$ will spread on the solid surface. In many cases, we never reach $\cos \theta=1$; then we can extrapolate the plot down to a value $\sigma_{\mathrm{lv}}=\sigma_{\mathrm{c}}$.

In 1963, Zisman [5] noticed that, for most low-energy solid surfaces and any homologous series of simple molecular liquids, the resulting curves (3) are linear. Thus, he established empirically, for a given low-energy solid surface, a linear relationship between the cosine of the contact angle $\theta$ and the surface tensions of a homologous series of simple molecular liquids, i.e. equation (2).

Zisman further generalized the concept of critical surface tension to high-energy surfaces like graphite, and to nonhomologous series of liquids. In the latter case, a strict linear relationship may not be seen but the data lie within a linear band that leads to an interval for $\sigma_{c}$.

\section{Brief review of theories of wetting under van der Waals forces}

In the theories of wetting, the intermolecular attractions play the most important role. The intermolecular attractions, which cause surface tension, arise from a variety of well-known intermolecular forces. Most of these forces, such as metallic bonding and hydrogen bonding, are a function of specific chemical nature. However, London dispersion forces exist in all types of matter and always give an attractive force between adjacent atoms or molecules, no matter how dissimilar their nature.

In order to deduce Zisman's equation (2) theoretically, we now review the theories of wetting under the condition that van der Waals forces are dominant, i.e. we deduce the explicit 
form of equation (3), as the mean step of the derivation of Zisman's equation (2), from Young's equation (1) by using the character of van der Waals forces.

It is known that the work of adhesion of a liquid and solid in contact, $W_{\mathrm{sl}}$, which is the work required to separate a unit area of the solid-liquid interface, is given by the Young-Dupré equation [11]

$$
W_{\mathrm{sl}}=\sigma_{\mathrm{sv}}+\sigma_{\mathrm{lv}}-\sigma_{\mathrm{sl}} \text {. }
$$

Combining equation (5) and Young's equation (1), we obtain

$$
\cos \theta=\frac{W_{\mathrm{s} 1}}{\sigma_{\mathrm{lv}}}-1
$$

Similarly, when we separate a unit area of the liquid-liquid interface, we obtain the work of cohesion of a liquid $W_{11}$ :

$$
W_{\mathrm{ll}}=2 \sigma_{\mathrm{lv}} \text {. }
$$

Combining equations (6) and (7), we obtain

$$
\cos \theta=\frac{2 W_{\mathrm{sl}}}{W_{\mathrm{ll}}}-1
$$

For simple molecular liquids and low-energy solid surfaces, van der Waals forces are dominant, so that we have approximately $[1,12]$

$$
W_{\mathrm{sl}}=k \alpha_{\mathrm{s}} \alpha_{1}
$$

and

$$
W_{11}=k \alpha_{1}^{2}
$$

where $\alpha_{\mathrm{s}}$ and $\alpha_{1}$ are the dielectric polarizabilities of the solid and liquid, respectively, and $k$ is an approximate constant depending upon the given solid and the given homologous series of liquids.

Substitution of equations (9) and (10) into equation (8) gives

$$
\cos \theta=2 \frac{\alpha_{\mathrm{s}}}{\alpha_{1}}-1
$$

Combining equations (7), (10) and (11), we get

$$
\cos \theta=\alpha_{\mathrm{s}} \sqrt{\frac{2 k}{\sigma_{\mathrm{lv}}}}-1 .
$$

Take $\theta=0$ in equation (12) and using equation (4), we obtain

$$
\sigma_{\mathrm{c}}=\frac{k}{2} \alpha_{\mathrm{s}}^{2}
$$

Combining equations (12) and (13), we get [1]

$$
\cos \theta=2 \sqrt{\frac{\sigma_{\mathrm{c}}}{\sigma_{\mathrm{lv}}}}-1 .
$$

We call this equation the Young-van der Waals equation, which, as will be shown in the next section, is the basis of Zisman's equation. 


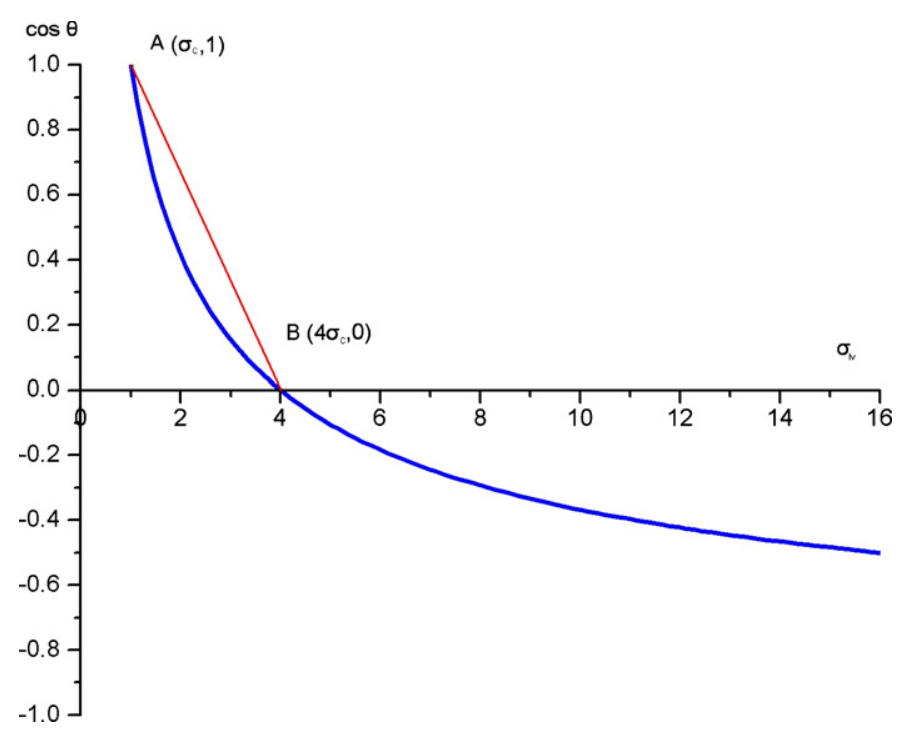

Figure 1. An illustration of the Young-van der Waals equation (14) and Zisman's equation (2).

(This figure is in colour only in the electronic version)

\section{Derivation of Zisman's equation}

For a nonlinear function, its figure is often helpful to us to be familiar with it. A figure of a function in the field of physics may also be helpful to understand the physical picture of the relevant physical problem. Thus, we plot the function of the theoretical equation (14) in figure 1.

From figure 1, we see that the segment of the curve of equation (14) in the region $\sigma_{\mathrm{c}} \leqslant \sigma_{\mathrm{lv}} \leqslant 4 \sigma_{\mathrm{c}}$ is the wetting section of the curve. It is obvious that the 'derivation of Zisman's equation' means to determine the empirical parameters $b$ and $\sigma_{\mathrm{c}}$ for Zisman's empirical equation (2) such that the wetting region of equation (2) is the same as that of the theoretical equation (14) (the first condition) and the difference between the straight line of equation (2) and the curve of equation (14) in the wetting region $\sigma_{\mathrm{c}} \leqslant \sigma_{\mathrm{lv}} \leqslant 4 \sigma_{\mathrm{c}}$ would be small (the second condition). The straight line satisfying the first condition must pass through both critical points $\left(A\left(\sigma_{\mathrm{c}}, 1\right) ; A\left(4 \sigma_{\mathrm{c}}, 0\right)\right)$, which determines uniquely the straight line

$$
\cos \theta=1+\frac{1}{3 \sigma_{\mathrm{c}}}\left(\sigma_{\mathrm{c}}-\sigma_{\mathrm{lv}}\right) \text {. }
$$

Identifying equation (15) with Zisman's equation (2), we obtain

$$
b=\frac{1}{3 \sigma_{\mathrm{c}}} \text {. }
$$

Substitution of equation (13) into equation (16) gives

$$
b=\frac{2}{3 k \alpha_{\mathrm{s}}^{2}} \text {. }
$$

The second condition requires that the line of equation (15) and the curve of equation (14) in figure 1 should be close to each other in the region $\sigma_{\mathrm{c}} \leqslant \sigma_{\mathrm{lv}} \leqslant 4 \sigma_{\mathrm{c}}$. Actually, we know from figure 1 that the second condition is satisfied approximately. 
Thus, we have shown theoretically that Zisman's empirical equation (2) is a linear approximation of the Young-van der Waals equation (14) in the wetting region $\sigma_{\mathrm{c}} \leqslant \sigma_{\mathrm{lv}} \leqslant 4 \sigma_{\mathrm{c}}$ and we have given the parameters $b$ and $\sigma_{c}$ in terms of the dielectric polarizabilities of the solid and liquids.

\section{Discussion}

From the above derivations of the Young-van der Waals equation (14) and Zisman's equation (2) we see that Young-van der Waals equation is valid under the precondition that van der Waals forces are dominant and relations (9) and (10) are valid approximately. Therefore, the Young-van der Waals equation (14) is an approximate equation, to say nothing of Zisman's equation (2).

For the cases where van der Waals forces are not dominant, both equations are no longer valid, and further experimental and theoretical studies of wetting of liquids on solid surfaces are being done. For example, Fowkes [7, 13-15] considered the surface tension $\sigma$ to be a measure of the attractive force between the surface layer and inner phase, and that such forces and their contribution to the free energy are additive. Therefore, the surface tension of liquid metals, polar liquids, hydrocarbons, low-energy solids and other solids is considered to be made up of independent additive terms:

$$
\sigma=\sigma^{\mathrm{d}}+\sigma^{\mathrm{h}}+\sigma^{\mathrm{m}}+\cdots
$$

where the $\sigma^{\mathrm{d}}$ term is attributed only to the London dispersion interactions, $\sigma^{\mathrm{h}}$ is due to hydrogen bonding, $\sigma^{\mathrm{m}}$ due to metallic bonding, etc. The readers who are interested in further particulars may refer to [7, 13-15].

\section{Conclusion}

Theories of wetting of liquids on solid surfaces under the condition that van der Waals force is dominant are briefly reviewed. We show theoretically that Zisman's empirical equation for wetting of liquids on solid surfaces is a linear approximation of the Young-van der Waals equation in the wetting region, and we have expressed the parameters $b$ and $\sigma_{\mathrm{c}}$ in Zisman's empirical equation in terms of the dielectric polarizabilities of the solid and liquids. The materials contained in this paper are suitable for physics teaching of wetting phenomena for undergraduate, graduate, general physicist, etc.

\section{Acknowledgments}

This work was supported by the National Natural Science Foundation of China (grant nos 10472128 and 10772189) and the Knowledge Innovation Program of Chinese Academy of Sciences.

\section{References}

[1] de Gennes P-G 1985 Rev. Mod. Phys. 57827

[2] Adamson A W 1990 Physical Chemistry of Surfaces 5th edn (New York: Wiley)

[3] de Gennes P-G, Brochard-Wyart F and Quere D 2004 Capillarity and Wetting Phenomena: Drops, Bubbles, Pearls, Waves (New York: Springer)

[4] Young T 1805 Phil. Trans. R. Soc. Lond. 9565

[5] Zisman A W 1963 Advances in Chemistry Series vol 43 (Washington: ACS)

[6] Dujardin E, Ebbesen T W, Krishnan A and Treacy M M J 1998 Adv. Mater. 101475 
[7] Sharma P K and Rao K H 2002 Adv. Colloid Interface Sci. 98341

[8] Dujardin E, Ebbesen T W and Krishnan A 1998 Adv. Mater. 101472

[9] Mattia D, Bau H H and Gogotsi Y 2006 Langmuir 221789

[10] Fox H W and Zisman A W 1952 J. Coll. Sci. 7109

[11] Kwok D Y and Neumann A W 1998 Prog. Colloid Polym. Sci. 109170

[12] Correia N T et al 1997 J. Colloid Interface Sci. 189361

[13] Fowkes F M 1962 J. Phys. Chem. 66382

[14] Fowkes F M 1964 Ind. Eng. Chem. 5640

[15] Fowkes F M, McCarthy D C and Mostafa M A 1980 J. Colloid Interface Sci. 78200 